\title{
Pedicled Versus Microvascular Free Flaps Used for Reconstruction of Oral Cavity Defects Following Cancer Ablative Surgery: A Prospective Comparative Study
}

Yasser Mohammed Seddeik Abd El Raheim Rayan ${ }^{* 1}$, Sherif Mohamed Khairallah ${ }^{2}$, Ahmed K Mousa ${ }^{3}$, Kamal abdel Aal Mohamed Hassanein Alsharkawy ${ }^{4}$, Mansour M Kabbash ${ }^{5}$

${ }^{1}$ Department of Maxillofacial Surgery, ${ }^{3}$ Department of Plastic Surgery, ${ }^{5}$ Department of General Surgery, Faculty of Medicine, Aswan University. ${ }^{2}$ Department of Oncosurgery, National Cancer Institute, Cairo University. ${ }^{4}$ Department of Maxillofacial, Head and Neck Surgery, Faculty of Medicine, Sohag University

*Corresponding Author: Yasser Mohammed Rayan, E-mail: superdoctor_2006@yahoo.com,

Mobile: (+20)01558494885

\begin{abstract}
Background: Oral cancer is currently a major global health issue. In developing countries, oral cavity cancer is estimated to be the third most common malignancy after cancer of the cervix and stomach. Surgical excision plays a major role in the treatment of oral cavity cancer patients.

Objective: The aim of the study was to compare between pedicled and free flaps used for oral cavity reconstruction after ablation of squamous cell carcinoma.

Patients and methods: This is a prospective comparative study that included 41 patients at National Cancer Institute (NCI) of Cairo University and Aswan University Hospital in the period from 2015 to 2018. Patients were selected from those who were attending the Outpatient Clinic of Head and Neck Surgery Unit at NCI, Cairo University and Aswan University Hospital.

Results: Detection of short-term functional outcome regarding the swallowing, speech and oral competence, Contralateral Submental Island Flap (CSMIF) followed by Radial Forearm Free Flap (RFFF) showed better functional results than other flaps. The cosmetic outcome was better in 50\% of CSMIF \& RFFF cases. The Thoracodorsal Artery Perforator Flap (TDAPF) cases showed a slightly higher care costs than other cases although in other specialized centers the care costs were found to be higher in RFFF cases than others.

Conclusion: Oncologic outcomes were better with CSMIF and cosmetic outcomes were better in CSMIF and RFFF cases. Relatively, TDAP flap cases were higher than others in the overall care costs.
\end{abstract}

Keywords: Pedicled flaps, Free flaps, Oral cavity reconstruction, Squamous cell carcinoma.

\section{INTRODUCTION}

The oral cavity is an aesthetically and functionally important area which serves form and many important functions ${ }^{(\mathbf{1})}$. The oral cavity has a complex anatomy and the reconstruction of defects in this area can be challenging. The goal of the reconstructive surgeon is to restore the integrity of the oral cavity and preserve important functions such as deglutition, articulation, and mastication ${ }^{(2)}$.

Surgical resection or ablation for treatment of patients with oral cancer may be performed through the transoral or transcervical approach. The approach used is determined by the location of the tumor, extent of the disease, treatment of the neck and planned reconstruction. The most commonly employed surgical approaches for resection of primary oral cancer are peroral, mandibulotomy, lower cheek flap approach, visor flap approach or upper cheek flap approach ${ }^{(3)}$.

Reconstruction should be tailored to the patient's ability to cope with a long operation and the risk of substantial morbidity. The reconstructive ladder starting from skin grafts and ending with free flaps may not always be able to be followed due to anatomical and functional requirements of the defects ${ }^{(4)}$.
Different pedicled and free tissue flaps had been reported in the reconstruction of tumor defects of head and neck region ${ }^{(5)}$. In our study, we discussed 4 types of flaps. Pectoralis Major MyoCutaneous Flap (PMMCF) has become the most frequently used regional flap and significantly improved the safety and functional outcome of head and neck surgery. The distinct advantage of PMMC lies in the fact that it is reliable, easy to harvest, can be raised in a short time, has minimal donor site morbidity, plenty of bulk, can be combined with other flaps such as free flap and can be used for single stage reconstruction of inner mucosal and/or outer skin defects in head and neck region ${ }^{(6)}$.

SMIF was first described by Martin and his colleagues in 1993 in their search for an ideal flap to restore facial defects with regard to color, shape and tissue texture ${ }^{(7)}$. Over the past two decades, this flap has gained acceptance as a reliable and easily available alternative in the reconstruction of oral cavity defects ${ }^{(\mathbf{8})}$.

\section{AIM OF THE WORK}

The aim of the study was to compare between pedicled and free flaps used for oral cavity 
reconstruction after ablation of squamous cell carcinoma (SCC).

\section{PATIENTS AND METHODS}

- Type, duration and location of the study: This is a prospective comparative study that included 41 patients at National Cancer Institute (NCI) of Cairo University and Aswan University Hospital in the period from 2015 to 2018.

- Sample size: The statistical power of the study was calculated using the results from Open Epi, version 3, open source calculator_SS proper to determine an adequate sample size for this study. With accuracy mode calculation and an effect size convention 7.9 for the independent samples t-test, with probability of 0.05 , provided $80 \%$ power for sample size of 20 patients in each group.

- Patient recruitments: Patients were selected from those who were attending the Outpatient Clinic of Head and Neck Surgery Unit at NCI, Cairo University and Aswan University Hospital.

- Study population: All patients diagnosed to have oral SCCs were considered as candidates for the study after meeting the inclusion criteria. Patients were classified into two groups;

Group (1); 20 cases were reconstructed by pedicled flaps;

- G1; 10 cases had reconstruction with PMMCF (Pectoralis Major MyoCutaneous Flap).

- G2; 10 cases had reconstruction with CSMIF (Contralateral Submental Island Flap).

Group (2); 21 cases were reconstructed with micro-vascular free flaps;

- G3; 11 cases had reconstruction with RFFF (Radial Forearm Free Flap).

- G4; 10 cases had reconstruction with TDAP (Thoracodorsal Artery Perforator Flap).

Inclusion criteria: Any patient diagnosed to have oral cavity cancer of SCC type.

Exclusion Criteria:

Patients who had Non- SCC types of oral cavity cancer.

Patients who had advanced disease; metastatic lesions away from the head and neck region.

Patients who had another primary lesion away from the head and neck region.

Patients who were medically unfit for surgery, major cardio-pulmonary diseases...etc.

\section{Methods:}

- All selected patients in this study were subjected to the following; full history taking and thorough clinical examination, certain preoperative clinical tests, investigations, postoperative care and follow-up strategies for a period of 12-24 months.

- All selected patients signed a written informed consent after explaining the benefits and hazards of each method of reconstruction.

Ethical consideration and Written informed consent:

An approval of the study was obtained from Aswan University academic and ethical committee. Every patient signed an informed written consent for acceptance of the operation.

\section{Intraoperative (surgical technique):}

- Operations were performed under general anesthesia by NCI Head and Neck Surgery Unit team and Aswan University Hospital team.

- Patient position ; mostly in supine position ( except when TDAP flap was harvested, the patient was put in right or left lateral decubitus position according to site from which the flap will be take.

- Urinary catheterization was done to evaluate the fluid output during the operation and postoperatively.

- Scrubbing by povidine-iodine and brushing superiorly from scalp hairline, curving with hairline to post-auricular region till nape and downwards involving face, neck and reach to upper chest (for SMIF or RFA flaps), umbilicus (for PMMCF), chest, umbilicus and flank (for TDAP flap) with scrubbing of forearm and hand (for RFFF).

Postoperative assessment and Follow-up strategies (ranged between 12-24 months):

*This period was for:

- Assessment of tumor ablation status with early detection of recurrence.

- Assessment of the chosen reconstructive flap and flap-related complications.

*This period was divided into early and late follow-up periods

Early follow-up; (for the $1^{\text {st }} 4$ weeks postoperatively)

- Postoperatively patients were kept in appropriate positions, avoid over-extension of the neck to minimize the tensile strength, pressure points or torsion on the pedicle (for pedicled flaps). 
- During this early follow-up period we followed;

- General condition of the patient.

- Operative time.

- Flap viability.

- Donor and recipient sites morbidity; bleeding, infection and healing process.

- Head and neck complications; nerves injury during ND, facial nerve injury and fistulae (orocutaneous salivary fistulae, chylous fistula ... etc).

Late follow-up; (from the beginning of $2^{\text {nd }}$ month to the end of the $24^{\text {th }}$ month postoperatively), during this late follow-up period we followed;

- Tumor ablation and early detection of locoregional recurrence.

- For assessment (follow up) of tumor ablation and early detection of recurrence:

- As long as about $70 \%$ of recurrences occur in the first year, we planned for six postoperative times for follow up scheduled as following; after 1 month, after 3 months, after 6 months, after 12 months, after 18 months and finally at end of 24 months.

- At each of these six times, the patient was assessed by;

- History taking (especially asking about any new complaints).

- Clinical examination (especially head and neck region).

- Radiological (U/S +/- CT or MRI) examination.

Postoperative care and follow-up aimed at: - Flap Monitoring for detection of postoperative flap-related and un-related complications;

- Flap-related complications :

- Viability of the flap; partial or total flap loss, necrosis or epidermolysis.

Post-operative Doppler examination ; done to check the viability of the flap, it was done by putting the probe on the pedicle of the flap, in case of pedicled flap surgery or on neck side where the anastomosis in the free flap surgery was carried out.

- Detection of flap failure main risk factors.

- Assessing the influence of irradiation on flap viability; ischemia and scar contractures.

- The probability of facial palsy during resection or $\mathrm{ND}$; as marginal branch injury as during harvesting of the SMIF.

- Oro- cutaneous or pharyngeo-cutaneous fistula;

- Does its formation related to usage of certain suturing materials (as Vicryl)?
- Usually it closes spontaneously, prophylactically the Ryle tube feeding was kept for not less or more than 10 days postoperatively.

- Neck skin color changes (as ecchymosis), dehiscence.

- Intra-oral flap dehiscence (i.e.; separation or loss of connection with oral mucosa).

- Donor site morbidities;

- Complications of wound healing, partial or total loss of skin graft, tendon exposure, reduced hand grip strength, visible donor site and possible poor cosmetic result, vascular (atherosclerosis, seldom), postoperative vascular compromise of hand (rarely); especially in RFFF.

- Abnormal sensation or even loss of function.

- Intra-oral hair growth in the flap (as in SMIF) harvested in male patients and was managed by epilation by YAG Laser Therapy.

- Flap surface mucosalization; as RFFF used to reconstruct palatal defects.

\section{Statistical analysis}

- All demographic characters, operative details, intraoperative and postoperative complications all were recorded.

All gathered data were verified, coded by the researcher and statistically analyzed using IBM-SPSS 21.0 (IBM-SPSS. Statistical Package for Social Science. Ver. 21. Standard deviation. Copyright $\odot$ SPSS Inc., 2011-2012. NY, USA.2012).

Descriptive statistics; Means and standard deviations were calculated.

Tests of significance;

- Chi-square test; was used to compare the difference in distribution of frequencies among different groups.

- For continuous variables with more than two categories, ANOVA test was calculated to test the mean differences of the data that follow normal distribution.

○ Independent sample Kruskal-Wallis was used to compare the median difference between groups that don't follow normal distribution.

- Post-hoc test was calculated using Bonferroni corrections.

- A probability value ( $\mathrm{p}$-value) is considered to be statistically significant if it is $\leq 0.05$.

\section{RESULTS}

On starting work preparation for this study, we collected data of 58 patients but unfortunately 17 patients were not included (as 2 
patients died on the first postoperative week, 10 patients died during the first 12 months postoperatively, 3 patients travelled abroad and were not be able to be involved in our study and 2 patients we failed to have a continuous contact with them as they preferred to continue their postoperative follow up in medical centers near their places of residency). Thus, only 41 patients were conducted on our study as those 41 patients successfully could complete the 24 months needed for the short-term period of postoperative follow up designed for patients who could coup with the strategy of this study. The results of the follow up of those 41 patients were discussed as mentioned below.

10 patients (24.4\%) had PMMCF reconstruction and called group 1, G1 (PMMCF) patients, 10 patients $(24.4 \%)$ had CSMIF reconstruction and called group 2, G2 (CSMIF) patients, 11 patients $(26.8 \%)$ had RFFF reconstruction and called group 3, G3 (RFFF) patients and 10 patients (24.4\%) had TDAP reconstruction and called group 4, G4 (TDAP) patients.

In G1 (PMMCF), the youngest patient had 37 years old and the oldest had 66 years old, in G2 (CSMIF), the youngest patient had 50 years old and the oldest had 78 years old, in G3 (RFFF), the youngest patient had 27 years old and the oldest had 68 years old and in G4 (TDAP), the youngest patient had 30 years old and the oldest had 72 years old. In the comparison between the flap groups, the mean age in patients of G2 (CSMIF) was higher than that in G4 (TDAP). In this study, the mean age of PMMCF patients was $53.50 \pm 9.1$ years, the mean age of CSMIF patients was $63.00 \pm 8.6$ years, the mean age of RFFF patients was 53.52 \pm 15.3 years and the mean age of TDAP patients was $46.60 \pm 15.5$ years (Table 1).

Regarding the sex parameter, in this study, there were 18 female patients $(43.9 \%)$ and 23 male patients (56.1\%). In G1 (PMMCF), there were 5 females (50\%) and 5 males (50\%), in G2 (CSMIF), there were 7 females $(70 \%)$ and 3 males (30\%), in G3 (RFFF), there were 4 females $(36.4 \%)$ and 7 males (63.6\%) and in G4 (TDAP), there were 2 females $(20 \%)$ and 8 males $(80 \%)$ (Table 1).

In this study there were 19 smoking patients $(46.3 \%)$ and 22 non-smoking patients (53.7\%). Regarding the smoking status among patients of G1 (PMMCF), there were $4(40 \%)$ smoking patients and $6(60 \%)$ non-smoking patients, in patients of G2 (CSMIF), there were 6 (60\%) smoking patients and $4(40 \%)$ nonsmoking patients, while in G3 (RFFF) there were 4 (36.4\%) smoking patients and 7 (63.6\%) nonsmoking patients and in patients of G4 (TDAP), there were $5(50 \%)$ smoking patients and $5(50 \%)$ non-smoking patients (Table 1).

Table (1): Socio-demographic differences between groups

\begin{tabular}{|c|c|c|c|c|c|}
\hline Parameter & $\begin{array}{c}\text { PMMCF (1) } \\
(n=10)\end{array}$ & $\begin{array}{c}\text { CSMIF (2) } \\
(n=10)\end{array}$ & $\begin{array}{c}\text { RFFF (3) } \\
(n=11)\end{array}$ & $\begin{array}{c}\text { TDAP (3) } \\
(n=10)\end{array}$ & P-value \\
\hline Age/years & $53.50 \pm 9.1$ & $63.00 \pm 8.6$ & $53.82 \pm 15.3$ & $46.60 \pm 15.5$ & \multirow{3}{*}{$\leq 0.053^{*}$} \\
\hline \multirow{2}{*}{ P-value** } & 1 vs. $2=0.103$ & 2 vs. $3=0.106$ & 3 vs. $4=0.201$ & 1 vs. $4=0.232$ & \\
\hline & 1 vs. $3=0.955$ & 2 vs. $4=0.006$ & & & \\
\hline \multicolumn{5}{|l|}{ Sex } & \multirow{3}{*}{$\leq 0.081 * * *$} \\
\hline • Female & $5(50 \%)$ & $7(70 \%)$ & $4(36.4 \%)$ & $2(20 \%)$ & \\
\hline$\bullet$ Male & $5(50 \%)$ & $3(30 \%)$ & $7(63.6 \%)$ & $8(80 \%)$ & \\
\hline \multicolumn{5}{|l|}{$\begin{array}{l}\text { Smoking } \\
\text { Status }\end{array}$} & \multirow{3}{*}{$\leq 0.628 * * *$} \\
\hline - Smoker & $4(40 \%)$ & $6(60 \%)$ & $4(36.4 \%)$ & $5(50 \%)$ & \\
\hline $\begin{array}{l}\text { - Non- } \\
\text { smoker }\end{array}$ & $6(60 \%)$ & $4(40 \%)$ & $7(63.6 \%)$ & $5(50 \%)$ & \\
\hline
\end{tabular}

*ANOVA test was used to compare the mean difference between groups.

***ost-hoc test with Bonferroni corrections.

$* * *$ Chi-square test was used to compare proportions between groups.

Regarding the clinical history of neoadjuvant therapy; 6 (14.63\%) patients had a positive history of radiotherapy, 2 (20\%) patients of G1 (PMMCF) and 4 (40\%) patients of G4 (TDAP) showed a positive history of radiotherapy, while no patient of G2 (CSMIF) or G3 (RFFF) had such history. 12 (29.27\%) patients had a positive history of chemotherapy; 6 (60\%) patients of G1 (PMMCF), 1 (10\%) patient of G1 (CSMIF), 1 (9.1\%) patient of G3 (RFFF) and 4(40\%) patients of G4 (TDAP) showed a positive history of chemotherapy (Table 2). 
ejhm.journals.ekb.eg

Table (2): Clinical history data differences between groups

\begin{tabular}{|c|c|c|c|c|c|}
\hline Parameter & $\begin{array}{l}\text { PMMCF(1) } \\
(n=10)\end{array}$ & $\begin{array}{l}\text { CSMIF } \\
(\mathrm{n}=10)\end{array}$ & $\begin{array}{l}\text { RFFF } \\
(n=11)\end{array}$ & $\begin{array}{l}\text { TDAP } \\
(n=10)\end{array}$ & P-value \\
\hline \multicolumn{6}{|l|}{ History of Operations } \\
\hline • Neck Surgery & $5(50 \%)$ & $2(20 \%)$ & $2(20 \%)$ & $5(50 \%)$ & $\leq 0.224 *$ \\
\hline \multicolumn{6}{|c|}{ Adjuvant Therapy History } \\
\hline - Radiotherapy & $2(20 \%)$ & $0(0 \%)$ & $0(0 \%)$ & $4(40 \%)$ & $\leq \mathbf{0 . 0 2 7} *$ \\
\hline - Chemotherapy & $6(60 \%)$ & $1(10 \%)$ & $1(9.1 \%)$ & $4(40 \%)$ & $\leq 0.028 *$ \\
\hline
\end{tabular}

*Chi-square test was used to compare proportions between groups.

Regarding the defect type, no mucosal (M) defect only needed to be reconstructed in all types of the flaps. The mucosa and muscle (MM) defects occurred in 8 (80\%) patients of G2 (CSMIF), $6(54.5 \%)$ patients of G3 (RFFF), 1(10\%) patient of G4 (TDAP) and there was no MM defect in G1 (PMMCF). In mucosa, muscle and bone (MMB) defects, there was defect in 3 (30\%) patients of G1 (PMMCF), 3 (27.3\%) patients of G3 (RFFF), 1 (10\%) patient of G4 (TDAP) and no MMB defects occurred in G2 (CSMIF). In mucosa, muscle and skin (MMS) defects, the defects ocuured in 5 (50\%) patients of G4 (TDAP), 2 (20\%) patients of G2 (CSMIF), 1 (10\%) patient of G1(PMMCF) and 1 (9.1\%) of G3 (RFFF). Regarding to composite defects (that involved mucosa, muscle, bone and skin), there was 6 (60\%) patients had defects in G1 (PMMCF), 3 (30\%) patients had defect in G4 (TDAP), 1 (9.1\%) patient had defect in G3 (RFFF) and there was no composite defect occurred in G2 (CSMIF) as shown in figure (1).

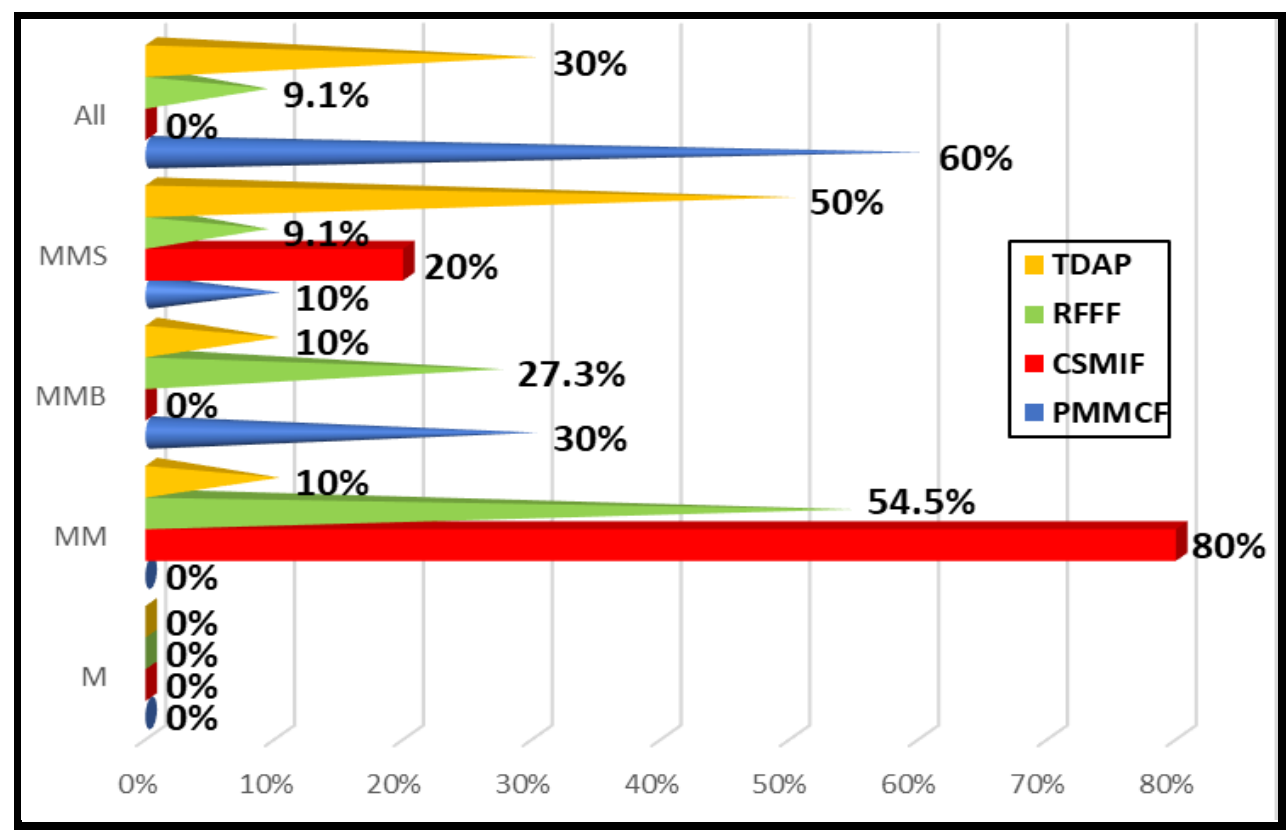

Fig. (1): Distribution of the studied sample according to defect type

Perioperative

histopathologic

examination [examination of the preoperative biopsy, the intraoperative frozen section examination and the postoperative full specimen examination (involving the examination of the resected tumor with the cervical fibro-fatty tissues involving the cervical lymph nodes and other neck structures according to the ND type)] revealed that our study cases of SCCs were graded as following (Fig. 2):

- Well differentiated SCC was detected in 4 patients (40\%) of G1 (PMMCF), in 5 patients
(50\%) of G2 (CSMIF), in 5 patients of G3 (RFFF) and in 3 patients (30\%) of G4 (TDAP).

- Mild undifferentiated SCC was not detected in any patient of the four flap groups.

- Moderatelly undifferentiated SCC was detected in 5 patients $(50 \%)$ of G1 (PMMCF), in 3 patients $(30 \%)$ of $\mathrm{G} 2$ (CSMIF), in 4 patients of G3 (RFFF) and in 6 patients $(60 \%)$ of G4 (TDAP).

- Poorly undifferentiated SCC was detected in 1 patient (10\%) of G1 (PMMCF), in 2 patients (20\%) of G2 (CSMIF), in 2 patients of G3 (RFFF) and in 1 patient (10\%) of G4 (TDAP). 


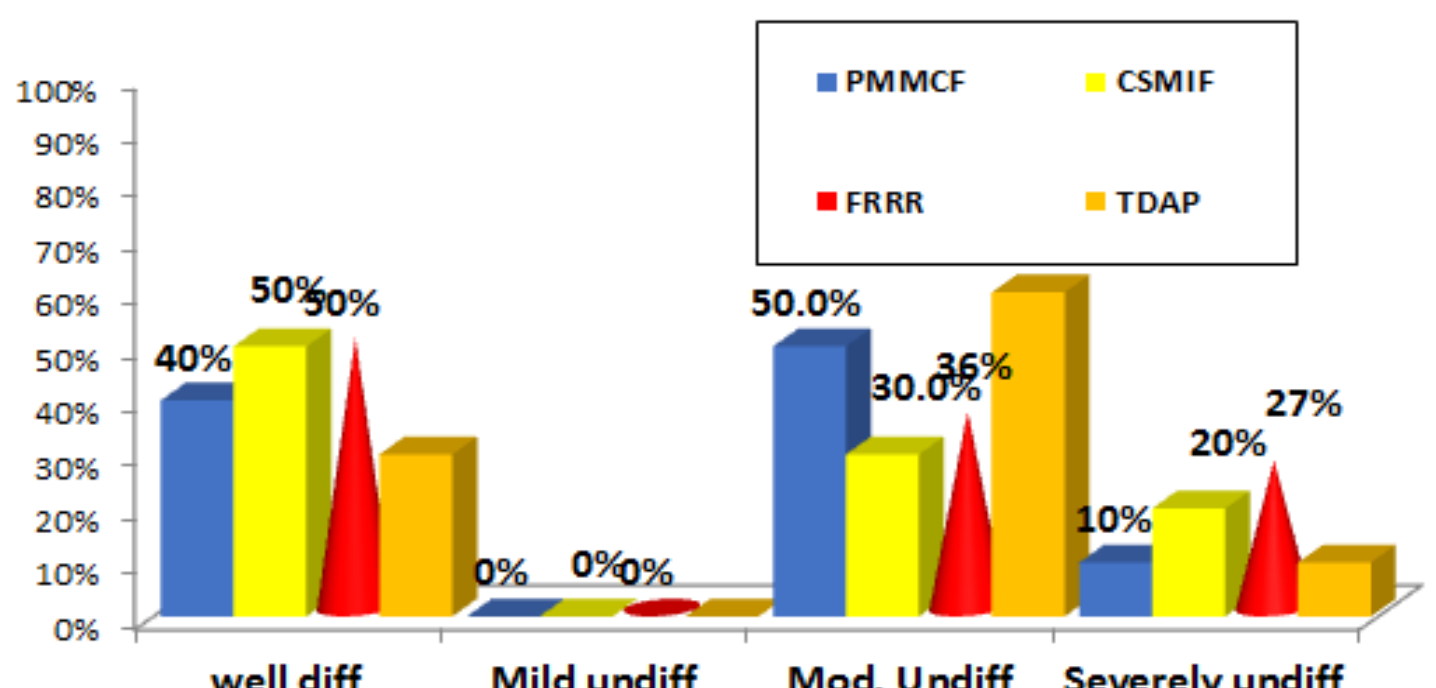

Fig. (2): Distribution of the studied sample according to the grade of SCC.

\section{Intraoperative complications:}

The mean operative time of PMMCF cases was $200 \pm 23.1$ minutes with a range $180-240$ minutes. The mean operative time of CSMIF cases was $199 \pm 20.2$ minutes with a range $180-250$ minutes. The mean operative time of RFFF cases was $220 \pm 19.2$ minutes with a range $200-250$ minutes. The mean operative time of TDAP cases was $212 \pm 24.9$ minutes with a range $180-250$ minutes (Table 3). According to the mean intraoperative blood loss in PMMCF and CSMIF cases was nearly equal $(760 \pm 68.6 \mathrm{ml}$ in PMMCF cases versus $760 \pm 61.8 \mathrm{ml}$ in CSMIF cases). The mean intraoperative blood loss in RFFF cases was $872.27 \pm 68.9 \mathrm{ml}$. The mean intraoperative blood loss in TDAP cases was $1020 \pm 48.4 \mathrm{ml}$ (Table 3 ). According to intraoperative flap-related complications, there was flap bulkiness occurred in 2 patients (20\%) of G1 (PMMCF) and 1 patient (10\%) of G4 (TDAP). Flap ischemia occurred in 2 patients (20\%) of G1 (PMMCF), 1 patient (10\%) of G2 (CSMIF), 1 patient (9.1\%) of G3 (RFFF) and 1 patient (10\%) of G4 (TDAP) (Table 3).

Table (3): Intra-operative sequence and complications differences between groups

\begin{tabular}{|c|c|c|c|c|c|}
\hline Parameter & $\begin{array}{c}\begin{array}{c}\text { PMMCF (1) } \\
(n=10)\end{array} \\
\end{array}$ & $\begin{array}{c}\text { CSMIF (2) } \\
(n=10)\end{array}$ & $\begin{array}{c}\text { RFFF (3) } \\
(n=11)\end{array}$ & $\begin{array}{c}\text { TDAP (3) } \\
(n=10)\end{array}$ & $P$-value \\
\hline $\begin{array}{l}\text { Operative } \\
\text { Time/min. }\end{array}$ & $200.00 \pm 23.1$ & $199.00 \pm 20.2$ & $220.00 \pm 19.2$ & $212.00 \pm 24.9$ & \multirow[b]{2}{*}{$\leq 0.084 *$} \\
\hline P-value** & $\begin{array}{c}1 \text { vs. } 2= \\
0.919 \\
1 \text { vs. } 3= \\
\mathbf{0 . 0 3 5}\end{array}$ & $\begin{array}{l}\mathbf{2} \text { vs. } 3=\mathbf{0 . 0 2 8} \\
2 \text { vs. } 4=0.192\end{array}$ & 3 vs. $4=0.358$ & 1 vs. $4=0.228$ & \\
\hline Blood Loss/ml & $760.00 \pm 68.6$ & $760.00 \pm 61.8$ & $872.27 \pm 68.9$ & $1020.00 \pm 48.4$ & \multirow[b]{2}{*}{$\leq 0.019 *$} \\
\hline P-value** & $\begin{array}{c}1 \text { vs. } 2= \\
1.000 \\
1 \text { vs. } 3= \\
0.208\end{array}$ & $\begin{array}{l}2 \text { vs. } 3=0.208 \\
2 \text { vs. } 4=0.103\end{array}$ & 3 vs. $4=0.103$ & 1 vs. $4=0.006$ & \\
\hline \multicolumn{6}{|c|}{ Intra-operative Complications } \\
\hline - No & $5(50 \%)$ & $9(90 \%)$ & $9(81.8 \%)$ & $9(90 \%)$ & \multirow{3}{*}{$\leq 0.019 * * *$} \\
\hline $\begin{array}{r}\text { Bulky } \\
\text { Flap }\end{array}$ & $2(20 \%)$ & $0(0 \%)$ & $0(0 \%)$ & $1(10 \%)$ & \\
\hline - Ischemia & $2(20 \%)$ & $1(10 \%)$ & $1(9.1 \%)$ & $1(10 \%)$ & \\
\hline
\end{tabular}

\footnotetext{
*ANOVA test was used to compare the mean difference between groups.

**Post-hoc test with Bonferroni corrections.

*** Chi-square test was used to compare proportions between groups.
} 


\section{Oncologic outcome:}

Loco-regional failure (LRF)/ Loco-regional recurrence (LRR) was estimated clinically and assessed radiologically by performing a postoperative CT and/or MRI.

There was a detectable clinical recurrence in 1 patient (10\%) in G1 (PMMCF), 1 patient in G3 (RFFF) and in 2 patients (20\%) in G4 (TDAP). There was an estimated radiological recurrence in 2 patients (20\%) in G1 (PMMCF), 3 patients in G3 (RFFF) and in 4 patients in G4 (TDAP) (Table 4).

Table (4): Late short-term (12-24 months postoperative) recurrence differences between groups.

\begin{tabular}{lccccc}
\hline Parameter & $\begin{array}{c}\text { PMMCF (1) } \\
(\mathbf{n = 1 0})\end{array}$ & $\begin{array}{c}\text { CSMIF (2) } \\
(\mathbf{n = 1 0})\end{array}$ & $\begin{array}{c}\text { RFFF (3) } \\
(\mathbf{n = 1 1})\end{array}$ & $\begin{array}{c}\text { TDAP (3) } \\
(\mathbf{n = 1 0})\end{array}$ & P-value \\
\hline $\begin{array}{l}\text { Clinical } \\
\text { LRF/LRR }\end{array}$ & $1(20 \%)$ & $0(0 \%)$ & $1(9.1 \%)$ & $2(20 \%)$ & $\leq \mathbf{0 . 1 8 0}$ \\
\hline $\begin{array}{l}\text { Radiological } \\
\text { LRF/LRR }\end{array}$ & $2(30 \%)$ & $0(0 \%)$ & $3(27.27 \%)$ & $4(40 \%)$ & $\leq \mathbf{0 . 1 8 0}$ \\
\hline
\end{tabular}

*Chi-square test was used to compare proportions between groups

\section{Evaluation of overall care costs:}

Regarding the difference in the mean overall care costs, the mean overall care cost in PMMCF cases was $136.13 \pm 31.4$ L.E., in CSMIF cases was $72.1 \pm 14$.2 L.E., in RFFF cases was $196.18 \pm 17.5$ L.E. and in TDAP cases was $199.5 \pm 17.4$ L.E. (Table 5).

Table (5): Overall care cost (per 1000 LE) differences between groups

\begin{tabular}{lccccc}
\hline Parameter & $\begin{array}{c}\text { PMMCF (1) } \\
(\mathbf{n = 1 0})\end{array}$ & $\begin{array}{c}\text { CSMIF (2) } \\
(\mathbf{n = 1 0})\end{array}$ & $\begin{array}{c}\text { RFFF (3) } \\
(\mathbf{n = 1 1})\end{array}$ & $\begin{array}{c}\text { TDAP (3) } \\
(\mathbf{n = 1 0})\end{array}$ & P-value \\
\hline Overall Care & $136.13 \pm 31.4$ & $72.10 \pm 14.2$ & $196.18 \pm 17.5$ & $199.50 \pm 17.4$ & \\
Cost & $\mathbf{1}$ vs. $\mathbf{2}=\mathbf{0 . 0 3 7}$ & $\mathbf{2}$ vs. $\mathbf{3}<\mathbf{0 . 0 0 1}$ & 3 vs. $4=0.904$ & $\mathbf{1}$ vs. $\mathbf{4}=\mathbf{0 . 0 3 9}$ & $\leq \mathbf{0 . 0 0 1 *}$ \\
P-value** & $\mathbf{1}$ vs. $\mathbf{3}=\mathbf{0 . 0 4 6}$ & $\mathbf{2}$ vs. $\mathbf{4}<\mathbf{0 . 0 0 1}$ & & & \\
\hline
\end{tabular}

*ANOVA test was used to compare the mean difference between groups

**Post-hoc test with Bonferroni corrections

\section{DISCUSSION}

In our study, all SMIF cases were harvested from the tumor contralateral side to stand on the hypothesis that CSMIF is better than ipsilateral submental island flap or not regarding or aiming at improvement of the oncologic outcome in our future patients not involved in this study.

RFFF is very useful flap for soft tissue intra-oral reconstruction. The vascular territory is reliable and offers significant versatility as either a fasciocutaneous, fascial, or osteocutaneous flap ${ }^{(9)}$. RFFF is relatively easy to raise from the volar region of the forearm. RFFF has pliable skin paddle, relatively hairless with less bulk spreads over shapes of oral cavity, and has good success

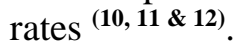

In our study regarding to PMMCF cases, the average age of the patients ranged from 37 to 66 years old (i.e. from $4^{\text {th }}$ to $7^{\text {th }}$ decades). There were 5 females (50\%), 5 males (50\%) 4 (40\%) smoking patients and $6(60 \%)$ non-smoking patients.

Regarding CSMIF cases, the average age of the patients ranged from 50 to 78 years old (i.e. from $6^{\text {th }}$ to $8^{\text {th }}$ decades). There were 7 females ( $\left.70 \%\right), 3$ males (30\%), $6(60 \%)$ smoking patients and 4
(40\%) non-smoking patients. In our study regarding RFFF cases, the average age of the patients ranged from 27 to 68 years old (i.e. from $3^{\text {th }}$ to $7^{\text {th }}$ decades). There were 4 females $(36.4 \%)$, 7 males (63.6\%), 4 (36.4\%) smoking patients and $7(63.6 \%)$ non-smoking patients. In our study regarding TDAP cases, the average age of the patients ranged from 30 to 72 years old (i.e. from $4^{\text {th }}$ to $8^{\text {th }}$ decades). There were 2 females $(20 \%), 8$ males (80\%), 5 (50\%) smoking patients and 5 (50\%) non-smoking patients.

According to the clinical history of neoadjuvant therapy, there was a significant difference between the four groups in which 4 patients (40\%) of G4 (TDAP), 2 patients (20\%) of G1 (PMMCF) showed a positive history of neoadjuvant radiotherapy while no patient of G2 (CSMIF) or G3 (RFFF) showed a positive history of neoadjuvant radiotherapy. 6 patients $(60 \%)$ of G1 (PMMCF), 1 patient (10\%) of G1 (CSMIF), 1 patient $(9.1 \%)$ of G3 (RFFF) and 4 patients (40\%) of G4 (TDAP) showed a positive history of neoadjuvant chemotherapy.

According to the site of oral cavity tumor, in our study the most common site of tumor was 
located at buccal (cheek) region in 21 patients (51.2\%) (gave a significant value about 0.029), followed by 11 patients had the tumor at tongue (also gave a significant value about 0.020), 8 patients had the tumor at gingival mucosa, 6 patients had the tumor at lip, 5 patients had the tumor at FOM and 3 patients had the tumor at the palate. These results are matching with a study done and documented that the commonest site of cancer was buccal mucosa ${ }^{(13)}$.

Regarding the defect in mucosa and muscle (MM), it occurred in 15 patients (36.6\%), followed by defects in mucosa, muscle, bone and skin (MMBS) that occurred in 10 patients (24.4\%), then defects in mucosa, muscle and skin (MMS) that occurred in 9 patients $(21.9 \%)$, then mucosa, muscle and bone (MMB) that occurred in 7 patients $(17.1 \%)$. Other studies reported different results, one of them showed that there was a defect in oral mucosal only in $65 \%$ of the patients ${ }^{(10)}$ and another demonstrated that the defect mainly was in the tongue and intraoral region ${ }^{(14)}$.

Regarding the TNM staging of oral cavity tumors in our study, tumors had T4 stage represented in 30 cases $(73.2 \%)$ and this gave a significant value of 0.001 . Another study reported that the major number of the patients $(43 \%)$ had T4 stage of the tumor ${ }^{(13)}$. Also, a study reported

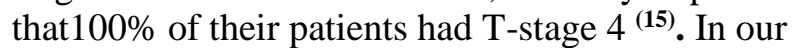
study tumors had $\mathrm{N}$-stage $\geq \mathrm{N}_{2}$ represented in 37 $(90.3 \%)$ cases. There was no tumors had M-Stage.

According to intraoperative flap-related complications, there was flap bulkiness that occurred only in 2 patients (20\%) of G1 (PMMCF) and 1 patient (10\%) in G4 (TDAP). Flap ischemia occurred in 2 patients (20\%) in G1 (PMMCF), 1 patient (10\%) in G2 (CSMIF), 1 patient (9.1\%) in G3 (RFFF) and 1 patient (10\%) in G4 (TDAP). These complications are not matching with Tornero et al. ${ }^{(16)}$ who reported that, they had not intraoperative complications. According to postoperative complications, the largest volume of blood loss occurred in drain with PMMCF, longest stay time at hospital occurred with TDAP, the longest period of ICU stay occurred with RFFF.

Locoregional recurrence or failure (LRR/LRF) in patients with oral SCC remains a problem and can affect their survival. In particular, local recurrence is a high potential risk factor for survival ${ }^{(17,18)}$. According to LRR/LRF in our cases, the highest rate of radiological recurrence was reported with TDAP ( $40 \%$ of the patients) followed by PMMCF group (30\% of the patients) then RFFF group (27.2\% of the patients) and there was no LRR/LRF reported with CSMIF. However, in report by Chow et al. ${ }^{\left({ }^{(9)}\right)}$ addressed these oncologic concerns by reviewing 10 cases of submental artery flap reconstruction after resection of aggressive oropharyngeal cancers. Three cancer recurrences were noted that were more likely related to the aggressive nature of the tumors than to the oncologic violation by the flap. Another study on CSMIF reported that $75 \%$ of the patients alive and free of disease, $20 \%$ of the patients had nodal relapse and 5\% of the patients had both local and distant relapse (20). Another study on CSMIF reported high recurrence rate by $44 \%$ of the patients in which $33 \%$ of the patients had cervical (nodal) recurrence ${ }^{(21)}$. Previous studies reported that local recurrence occurred in $6.9-22.0 \%{ }^{(22)}$.

A study reported that all their patients underwent dental rehabilitation to improve chewing and cosmosis 3 months postoperatively. Presence of the flap did affect neither the stability nor the installation of the prosthesis ${ }^{(\mathbf{2 3})}$. Also there was studies reported the disadvantages of the RFFF, which was cosmetic appearance of the donor site especially in females and sometimes delayed donor site healing, this complication occurred in up to $33 \%$ of patients ${ }^{\text {(24). }}$.

Regarding the difference in the mean overall care costs was statistically insignificant, the mean overall care cost in PMMCF cases was $136.13 \pm$ 31.4 L.E., in CSMIF cases was $72.1 \pm 14.2$ L.E., in RFFF cases was $196.18 \pm$ 17.5 L.E. and in TDAP cases was $199.5 \pm 17.4$ L.E.

A study to detect morbidity and cost differences between pedicled and free flaps used in oral and oropharyngeal cancer reported that no statistical difference in care costs of 36 patient underwent PMMCF reconstruction compared to 127 patient had RFFF reconstruction (25). Another studies reported the care costs in head and neck reconstruction with PMMCF to be less expensive than FRRR.

\section{CONCLUSION}

The chosen 4 flaps for this study were applicable to be used for post ablative oral cavity reconstruction, but TDAP flaps were found to be 2 times more liable for risk of complications than PMMCF flaps while RFFF flaps were found to be 1.3 times more liable for risk of complications than PMMCF flaps. CSMIF flaps showed a 58\% less liability for developing clinically or radiologically detectable LRF/LRR and showed no mortalities. LRF/LRR was more with TDAP flaps (2.6 times more risk or liability for LRF/LRR than PMMCF) followed by RFFF flaps (1.2 times more risk or liability for LRF/LRR than PMMCF).

Our flaps were equal in suitability for defect coverage $(50-60 \%)$. Functional outcomes were better with CSMIF then with RFFF, and these outcomes were worse with TDAP. Oncologic 
outcomes were better with CSMIF and cosmetic outcomes were better in CSMIF and RFFF cases. Relatively, TDAP flap cases were higher than others in the overall care costs.

\section{REFERENCES}

1. Gandhi A, Ramamurthy $\mathbf{R}$, Sganmugan $\mathbf{S}$ (2017): Functional assessment after oral cancer resection is objective assessment mandatory. Indian J Sci Res., 7 (2): 125-129.

2. Rigby MH, Taylor SM (2013): Soft tissue reconstruction of the oral cavity: a review of current options. Curr Opin Otolaryngol Head Neck Surg., 21 (4): 311- 7.

3. McGregor AD, MacDonald DG (1989): Patterns of spread of squamous cell carcinoma within the mandible. Head Neck, 11: 457-461.

4. Yadav P (2007): Reconstructive surgery in oral cancers. Indian Journal of Plastic Surgery, 40 (12): 22-27.

5. Neligan PC, Nithya C, Pandey M et al. (2003): Patterns of cervical metastasis from carcinoma of the oral tongue. World J Surg Oncol., 1: 10-3.

6. Ariyan S (1979): The pectoralis major myocutaneous flap. A versatile flap for reconstruction in the head and neck. Plastic and Reconstructive Surgery, 63 (1): 73-81.

7. Martin D, Pascal JF, Baudet J et al. (1993): The submental island flap: a new donor site. Anatomy and clinical applications as a free or pedicled flap. Plast Reconstr Surg., 92: 867-873.

8. Abouchadi A, Capon-Degardin N, Patenôtre P et al. (2007): The submental flap in facial reconstruction: advantages and limitations. J Oral Maxillofac Surg., 65 (5): 863-9.

9. Sader C, Hart RD, Trites JR et al. (2010): The communicating vein in the radial forearm free flap. Plast Reconstr Surg., 126: 105- 107.

10. Gupta A, Agrawal G, Tiwari S et al. (2015): Pectoralis major myocutaneous flap in head and neck reconstruction: an interesting experience from central India regional cancer center. International Journal of Research in Medical Sciences, 3 (11): 3065-3068.

11. Jeremić JV, Nikolić ŽS (2015): Versatility of Radial Forearm Free Flap for Intraoral Reconstruction. Srp Arh Celok Lek., 143 (5-6): 256-60.

12. Umakanth GB, Manish G, Srikanth K et al. (2016): A Study of Radial Forearm Free Flap in Intra Oral Defects following Resection of Oral Cavity Cancers. Journal of Dental and Medical Sciences, 15 (07): 23-26.

13. Chakrabarti S, Pancholi M, Mehta DY et al. (2015): Reconstruction in oral malignancy:
Factors affecting morbidity of variousprocedures. Ann Maxillofac Surg., 5 (2): 191-198.

14. Zhang YX, Xi W, Lazzeri D et al. (2015): Bipaddle radial forearm flap for head and neck reconstruction. J Craniofac Surg., 26 (2): 350-3.

15. Pradhan P, Samal S, Preetam C et al. (2018): Pectoralis Major Myocutaneous Flap Reconstruction for the Mandibular Defects in Advanced Oral Cavity Malignancies: A Retrospective Study of 30 Cases. Indian J Otolaryngol Head Neck Surg., 70 (3): 415-420.

16. Tornero J, Cruz-Toro $P$, Farré A et al. (2014): Free radial forearm flap in head and neck: our experience. Acta Otorrinolaringol Esp., 65 (1): 27-32.

17. Brandwein-Gensler M, Teixeira MS, Lewis CM et al. (2005): Oral squamous cell carcinoma. Histologic risk assessment, but not margin status, is strongly predictive of local disease-free and overall survival. Am J Surg Pathol., 29: 167-178.

18. Yanamoto $S$, Yamada $S$, Takahashi $H$ et al. (2012): Clinicopathological risk factors for local recurrence in oral squamous cell carcinoma. Int J Oral Maxillofac Surg., 41: 1195-1200.

19. Chow TL, Chan TT, Chow TK et al. (2007): Reconstruction with submental flap for aggressive orofacial cancer. Plast Reconstr Surg., 120 (2): 431-6

20. Amin AA, Sakkary MA, Khalil AA et al. (2011): The submental flap for oral cavity reconstruction: extended indications and technical refinements. Head Neck Oncol.. 20 (3): 51-55.

21. Cariati P, Cabello SA, Marin FAB et al. (2018): Is submental flap safe for the oncological reconstruction of the oral cavity? J Stomatol Oral Maxillofac Surg., 119 (4): 284- 287.

22. Kernohan MD, Clark JR, Gao K et al. (2010): Predicting the prognosis of oral squamous cell carcinoma after first recurrence. Arch Otolaryngol Head Neck Surg., 136: 1235-1239.

23. Elsherbiny M, Mebed A, Mebed H (2008): Microvascular radial forearm fasciocutaneous free flap for palatomaxillary reconstruction following malignant tumor resection. J Egypt Natl Canc Inst., 20 (1): 90-7.

24. Dufloa S, Liefb F, Parisa J et al. (2005): Microvascular radial forearm fasciocutaneous free flap in hard palate reconstruction. EJSO., 31: 784-791.

25. Smeele LE, Goldstein D, Tsai V et al. (2006): Morbidity and cost differences between free flap reconstruction and pedicled flap reconstruction in oral and oropharyngeal cancer: Matched control study. J Otolaryngol., 35 (2): 102-7. 